\author{
K. Rossing • P. K. Christensen • P. Hovind • \\ H.-H. Parving
}

\title{
Remission of nephrotic-range albuminuria reduces risk of end-stage renal disease and improves survival in type 2 diabetic patients
}

Received: 10 February 2005 / Accepted: 16 June 2005 / Published online: 17 September 2005

(C) Springer-Verlag 2005

\begin{abstract}
Aims/hypothesis: We evaluated the impact of remission of nephrotic-range albuminuria $(>2500 \mathrm{mg} / 24 \mathrm{~h}$ ) (NRA) on end-stage renal disease (ESRD) and mortality in type 2 diabetic patients with nephropathy. Methods: This was a follow-up observational study involving all 79 patients ( $35 \%$; 62 men, 17 women) with NRA from a cohort of type 2 diabetic patients with nephropathy that was followed for at least 3 years at the Steno Diabetes Center $(n=227)$. Patients were followed from the onset of NRA until death or January 2005 . The mean age $( \pm \mathrm{SD})$ was $60 \pm 8$ years and known diabetes duration was $14 \pm 7$ years. Remission of NRA was defined as sustained albuminuria $<600 \mathrm{mg} / 24 \mathrm{~h}$ for at least 1 year. Results: The duration of follow-up after onset of NRA was 6.5 years (range 2-20 years). Remission was induced in $20(25 \%)$ of the patients, all treated with ACE inhibitors or angiotensin-II receptor blockers. Remission lasted 4.1 years (range $1-10$ years) and only three patients relapsed. At the end of follow-up, only $30 \%$ (two ESRD and four deaths) of the 20 patients with remission had reached the composite endpoint of ESRD or death, in contrast to $66 \%$ (16 ESRD and 23 deaths) of the 59 patients without remission $(p<0.01)$. Cox regression analysis revealed that remission was associated with a risk reduction of $67 \%(95 \%$ CI $10-87)$ for reaching the composite endpoint of ESRD or death and of $69 \%(95 \%$ CI $21-88 \%$ ) for death alone. Male sex, greater age and systolic blood pressure at onset of NRA were also independently associated with an increased risk of ESRD and death. Conclusions/interpretation: Aggressive antihypertensive treatment can lead to long-term remission of NRA in a sizeable proportion of patients with type 2
\end{abstract}

K. Rossing $(\bowtie) \cdot$ P. K. Christensen $\cdot$ P. Hovind $\cdot$ H.-H. Parving Steno Diabetes Center,

Niels Steensens Vej 2,

2820 Gentofte, Denmark

e-mail: krossing@dadlnet.dk

Tel.: +45-4-4439205

Fax: $+45-4-4438232$

H.-H. Parving

Faculty of Health Science, Aarhus University,

Aarhus, Denmark diabetes. Such remission is associated with a slower progression of nephropathy and substantially improved survival.

Keywords Albuminuria - Antihypertensive treatment . Blood pressure $\cdot$ Diabetic nephropathy $\cdot$ End-stage renal disease $\cdot$ Mortality $\cdot$ Nephrotic-range albuminuria $\cdot$ Type 2 diabetes

Abbreviations ACE-I: ACE inhibition - ARB:

Angiotensin II receptor blocker - ESRD: End-stage renal disease - NRA: Nephrotic-range albuminuria - RAAS: Renin-angiotensin-aldosterone system

\section{Introduction}

Diabetic nephropathy has traditionally been regarded as a progressive and irreversible disease [1]. Before the introduction of antihypertensive agents as a key component in the treatment of the disease in the late 1970s, the clinical course of diabetic nephropathy was characterised by a continuous increase in blood pressure and albuminuria, and an inevitable decline in GFR of approximately $10-15 \mathrm{ml} \mathrm{min}^{-1}$ year $^{-1}$ [2]. Consequently, end-stage renal disease (ESRD) and death ensued a few years after the onset of diabetic nephropathy. A particularly poor prognosis has for many years been described for the subset of patients with nephrotic-range albuminuria (NRA, albuminuria $>2500 \mathrm{mg} / 24 \mathrm{~h}$ ) [3]. The degree of albuminuria is closely correlated to renal and cardiovascular outcome [3-6] and patients with NRA have the most severe renal structural damage, the greatest risk of ESRD, and the shortest survival time [1-3].

During the past three decades, substantial improvements have been achieved in the treatment of diabetic nephropathy, primarily through the implementation of early and aggressive blood-pressure-lowering treatment, which slows down the loss of renal function, reducing the risk of ESRD, as well as cardiovascular morbidity and mortality [7-10]. Furthermore, the widely held view that onset of NRA in patients with diabetes heralds the onset of inexorable pro- 
gression to ESRD has been challenged in several studies of patients with type 1 diabetes, which demonstrated that sustained remission of NRA (defined as a reduction of albuminuria from $2500 \mathrm{mg} / 24 \mathrm{~h}$ to $<600 \mathrm{mg} / 24 \mathrm{~h}$ ) is feasible in a large proportion of patients by using aggressive antihypertensive treatment, and is associated with substantial improvements in renal and cardiovascular outcome [11-13]. However, there are no data regarding the incidence of NRA and the possibility of obtaining remission for patients with type 2 diabetes.

We therefore evaluated the incidence of NRA and the frequency of remission in a large cohort of patients with type 2 diabetes who were followed at the Steno Diabetes Center. We also evaluated the impact of remission on the long-term course of renal disease and all-cause mortality.

\section{Subjects and methods}

Subjects Subjects were from a previously described cohort of patients with type 2 diabetes and nephropathy, who were followed for at least 3 years at the Steno Diabetes Center $(n=227)$ [14]. In the present observational followup study, all of the above patients who developed NRA $(n=79)$ were included, and followed from the onset of NRA either until death, or until January 2005.

Type 2 diabetes was diagnosed in patients treated by diet alone or by diet combined with oral hypoglycaemic agents; in insulin-treated patients with a BMI above normal ( $\geq 25 \mathrm{~kg} / \mathrm{m}^{2}$ in women, $\geq 27 \mathrm{~kg} / \mathrm{m}^{2}$ in men) at the time of diagnosis and in whom diabetes onset was after the age of 40 years; or in insulin-treated patients with a normal BMI if a glucagon-stimulated C-peptide value was $\geq 0.60 \mathrm{pmol} / 1$.

Diabetic nephropathy was diagnosed clinically in patients with: (1) albuminuria $>300 \mathrm{mg} / 24 \mathrm{~h}$ in at least two out of three consecutive samples; (2) diabetic retinopathy; and (3) no clinical or laboratory evidence of non-diabetic renal disease [2]. In all patients with abscence of diabetic retinopathy, the diagnosis of diabetic nephropathy was established by a renal biopsy showing diabetic glomerulosclerosis.

NRA was defined as persisting albuminuria $>2500 \mathrm{mg} / 24 \mathrm{~h}$ (which corresponds to total proteinuria $>3500 \mathrm{mg} / 24 \mathrm{~h}$ ) in at least two out of three consecutive 24-h urine collections. Remission of NRA was defined as a reduction in albuminuria from NRA to $<600 \mathrm{mg} / 24 \mathrm{~h}$, sustained for at least 1 year during follow-up. These definitions of NRA and remission of NRA are in accordance with previous published studies [11, 12].

During the observation period, patients were seen at the Steno Diabetes Center for routine visits three or four times a year. Body weight, postprandial blood glucose, $\mathrm{HbA}_{1} \mathrm{c}$, blood pressure and 24-h albuminuria were determined at each visit; GFR, serum creatinine and haemoglobin were measured once a year; and cholesterol was measured at least every second year. No sodium or protein restriction was applied during the study. During follow-up, the treatment goal for $\mathrm{HbA}_{1} \mathrm{c}$ was $7.5 \%$ as recommended by the Danish Medical Association. Arterial hypertension was diagnosed and treated according to the World Health Organization's criteria $(\geq 160 / 95 \mathrm{mmHg})$ until 1995 and thereafter according to the American Diabetes Association's criteria $(\geq 140 / 90 \mathrm{mmHg})[15]$. Until 2001, no specific class of antihypertensive agents was recommended. The most commonly prescribed agents included diuretics, ACE inhibitors (ACE-I), calcium-channel blockers and beta-blockers. After 2001, agents blocking the renin-angiotensin-aldosterone system (RAAS) were recommended as the initial drug of choice $[9,10,16]$. At the Steno Diabetes Center, low-dose aspirin and statins were not used systematically until 2002 in patients with type 2 diabetes with macroalbuminuria.

Materials and methods ESRD was defined as serum creatinine $>500 \mu \mathrm{mol} / 1$, the need for dialysis or renal transplantation. In all patients included in the study, we obtained information on survival status/date of death in January 2005 from the Danish death registry. Death certificates were obtained to establish the cause of death. In patients who died with ESRD, the cause of death was coded as ESRD irrespective of the cause of death on the death certificate.

Diabetic retinopathy was assessed by direct ophthalmoscopy until 1988, thereafter by fundus photography, both after pupillary dilatation. The degree of retinopathy was classified as nil, simplex or proliferative. Diabetic maculopathy was graded as simplex retinopathy.

GFR was measured after a single intravenous injection of ${ }^{51} \mathrm{Cr}-\mathrm{EDTA}(3.7 \mathrm{MBq})$ at $08.30 \mathrm{~h}$ by following the plasma clearance of the tracer for $4 \mathrm{~h}$ [17]. The results were standardised for $1.73 \mathrm{~m}^{2}$ body surface area. The surface area calculated at the initial GFR measurement was used to standardise all subsequent GFR measurements for a body surface area of $1.73 \mathrm{~m}^{2}$.

$\mathrm{HbA}_{1} \mathrm{c}$, serum creatinine, cholesterol and haemoglobin were measured using standard laboratory techniques with unchanged reference intervals over the study period, as described in detail previously [14].

Albuminuria was quantified by radioimmunoassay from 1983 to 1990 (sensitivity $0.5 \mathrm{mg} / \mathrm{l}$, coefficient of variation 9\%), [18] and from 1990 to 1997 by enzyme immunoassay (sensitivity $1.1 \mathrm{mg} / \mathrm{l}$, coefficient of variation 8\%) [19]. A close correlation between radioimmunoassay and enzyme immunoassay $(r=0.99)$ was documented before changing the methods. From 1997, the DAKO Turbidimetric method was used to measure urinary albumin excretion rate. This method is closely correlated with enzyme immunoassay $(r=0.99)$ and has a coefficient of variation of $5 \%$.

Blood pressure was measured in the sitting position after approximately 5-10-min rest using a standard mercury sphygmomanometer and an appropriate cuff size.

Smoking history was assessed from the patient's records. Patients were classified as smokers if they smoked more than one cigarette per day. The study was approved by the local ethics committee and was conducted in accordance with the Helsinki declaration.

Statistical analysis Results are expressed as mean $( \pm \mathrm{SE})$ unless otherwise stated. Albuminuria was logarithmically 
transformed before analysis because of its skewed distribution and is given as geometric mean with $95 \%$ confidence intervals. Baseline values of clinical variables were calculated as mean values during the first year after onset of NRA, and clinical values at end of follow-up were calculated as mean values during the last year of observation. Comparisons were performed with Student's $t$-test and Fisher's exact test. A Cox proportional hazard regression analysis with remission as endpoint was performed to evaluate baseline predictors of subsequent remission. In this analysis, time from NRA was used as timescale. Age, sex, and baseline albuminuria, $\mathrm{HbA}_{1} \mathrm{c}$ and systolic blood pressure were included as covariates. Logistic regression with backwards selection was performed to evaluate factors that were associated with remission of NRA during follow-up. To evaluate baseline predictors and the impact of remission on ESRD and death, two other Cox proportional hazard regression analyses were performed: one with the composite endpoint of ESRD or death, the other with only death as endpoint. In both analyses, the time since NRA was used as the timescale. Based on a previous study of risk factors for progression of renal disease and mortality in type 2 diabetes at the Steno Diabetes Center [14], we decided a priori to include the following fixed baseline covariates in both models: age, sex, $\mathrm{HbA}_{1} \mathrm{c}$ and systolic blood pressure. Remission of NRA was entered as a time-dependent covariate with value 0 up to the time of remission and after that a value of 1 . For patients with onset of NRA within the first 3 years after onset of nephropathy, delayed entry was used, because the inclusion criterion required at least 3 years of follow-up with nephropathy. Linear regression analysis by leastsquares method was used to determine the GFR for each patient. Statistical analyses were performed using SPPS 12.0 (SPSS, Chicago, IL, USA) and using the freely available software R 2.0.1 (http://www.r-project.org). A p-value of less than 0.05 was considered statistically significant.

\section{Results}

Occurrence of NRA and remission

NRA occurred in 79 (35\%) of the 227 type 2 diabetic patients with nephropathy during a mean follow-up period of 6.5 years (range 3-20 years). The 79 patients with NRA were followed from the onset of their NRA until development of ESRD, death, or January 2005. The mean duration of follow-up was 6.5 years (range 2-20 years). Remission of NRA was obtained in $20(25 \%)$ of the 79 patients, on average 2.7 years $(0.5-7$ years $)$ after the onset of NRA, and remission was sustained for 4.1 years (1-13 years). Of the 20 patients with remission, 17 remained in remission until the end of follow-up, with a mean total follow-up period of 7.3 years (3-13 years) and a mean duration of remission of 4.5 years (1-10 years). Three patients who were followed for between 6 and 10 years relapsed after one to three years.
Table 1 Baseline characteristics of 79 type 2 diabetic patients with NRA stratified to whether or not remission was obtained during follow-up

\footnotetext{
${ }^{\mathrm{a}}$ Mean values (SE)

${ }^{\mathrm{b}}$ Geometric mean $(95 \% \mathrm{CI})$ during the first year after onset of NRA
}

\begin{tabular}{|c|c|c|c|}
\hline & \multicolumn{2}{|l|}{ Baseline } & \multirow[t]{2}{*}{$p$-value } \\
\hline & Remission, $n=20$ & No remission, $n=59$ & \\
\hline Age (years) ${ }^{a}$ & $60(1)$ & $60(2)$ & 0.76 \\
\hline Male sex, $n(\%)$ & $15(75)$ & $47(80)$ & 0.76 \\
\hline Known diabetes duration (years) & $14(1)$ & $14(1)$ & 0.65 \\
\hline $\operatorname{BMI}\left(\mathrm{kg} / \mathrm{m}^{2}\right)^{\mathrm{a}}$ & $30.8(0.9)$ & $28.9(0.6)$ & 0.1 \\
\hline \multicolumn{4}{|l|}{ Diabetic retinopathy, $n(\%)$} \\
\hline None & $5(25)$ & $7(12)$ & 0.37 \\
\hline Simplex & $10(50)$ & $35(59)$ & \\
\hline Proliferative & $5(25)$ & $17(29)$ & \\
\hline History of ischaemic heart disease, $n(\%)$ & $3(15)$ & $10(17)$ & 1.0 \\
\hline History of stroke, $n(\%)$ & $2(10)$ & $12(20)$ & 0.50 \\
\hline Smoking, $n(\%)$ & $8(40)$ & $22(37)$ & 0.52 \\
\hline \multicolumn{4}{|l|}{ Hypoglycaemic treatment, $n(\%)$} \\
\hline Diet alone & $3(15)$ & $3(5)$ & 0.26 \\
\hline Oral hypoglycaemic agents & $5(25)$ & $26(44)$ & \\
\hline Insulin & $8(40)$ & $23(39)$ & \\
\hline Oral hypoglycaemic agents+insulin & $4(20)$ & $7(12)$ & \\
\hline $\operatorname{GFR}\left(\mathrm{ml} \mathrm{min} \operatorname{mon}^{-1} 1.73 \mathrm{~m}^{-2}\right)^{\mathrm{a}}$ & $73(5)$ & $66(4)$ & 0.7 \\
\hline Albuminuria $(\mathrm{mg} / 24 \mathrm{~h})^{\mathrm{b}}$ & $2802(2541-3092)$ & $3081(2881-3295)$ & 0.17 \\
\hline \multicolumn{4}{|l|}{ Blood pressure $(\mathrm{mmHg})^{\mathrm{a}}$} \\
\hline Systolic & $161(4)$ & $162(2)$ & 0.9 \\
\hline Diastolic & $84(3)$ & $85(1)$ & 0.9 \\
\hline $\mathrm{HbA}_{1} \mathrm{c}(\%)^{\mathrm{a}}$ & $8.9(0.4)$ & $8.5(0.2)$ & 0.2 \\
\hline Serum cholesterol $(\mathrm{mmol} / \mathrm{l})^{\mathrm{a}}$ & $5.9(0.4)$ & $6.2(0.2)$ & 0.3 \\
\hline Haemoglobin $(\mathrm{mmol} / \mathrm{l})^{\mathrm{a}}$ & $8.3(0.3)$ & $8.6(0.3)$ & 0.3 \\
\hline
\end{tabular}


Table 2 Characteristics during follow-up in 79 patients with type 2 diabetes and NRA showing data for patients with and without remission

\begin{tabular}{|c|c|c|c|}
\hline & Remission $(n=20)$ & No remission $(n=59)$ & $p$ value \\
\hline Duration of follow-up (years) & $7.5(2-13)$ & $6.2(2-20)$ & 0.12 \\
\hline Albuminuria $(\mathrm{mg} / 24 \mathrm{~h})^{\mathrm{a}}$ & $494(359-680)$ & $2677(2364-3031)$ & - \\
\hline \multicolumn{4}{|l|}{ Blood pressure $(\mathrm{mmHg})$} \\
\hline Systolic & $148(4)$ & $160(2)$ & 0.01 \\
\hline Diastolic & $76(2)$ & $80(1)$ & 0.09 \\
\hline Rate of decline in GFR $\left(\mathrm{ml} \mathrm{min}^{-1} \text { year }{ }^{-1}\right)^{\mathrm{b}}$ & $5.4(1.0)$ & $8.4(1.1)$ & 0.08 \\
\hline $\mathrm{HbA}_{1} \mathrm{c}(\%)$ & $8.7(0.7)$ & $8.4(0.2)$ & 0.42 \\
\hline Serum cholesterol $(\mathrm{mmol} / \mathrm{l})$ & $4.9(0.3)$ & $5.8(0.2)$ & 0.02 \\
\hline Haemoglobin $(\mathrm{mmol} / \mathrm{l})$ & $8.0(0.2)$ & $8.1(0.1)$ & 0.68 \\
\hline
\end{tabular}

Values are mean (SE) or ${ }^{\text {a }}$ geometric mean $(95 \% \mathrm{CI})$ during the entire follow-up period

${ }^{\mathrm{b}}$ Includes all 55 patients (16 with remission and 39 without remission) who had at least 3 years with annual GFR measurements during the observation period, whereas the remaining 24 patients with less than three GFR measurements were excluded

Characteristics of patients at baseline and during follow-up

At the onset of NRA, patients with and without subsequent remission were similar with respect to age, sex, degree of diabetic retinopathy, previous cardiovascular disease, percentage of smokers, GFR, albuminuria, arterial blood pressure, $\mathrm{HbA}_{1} \mathrm{c}$ and serum cholesterol levels (Table 1). A Cox regression analysis revealed no significant impact of sex, calendar year of NRA onset, or baseline age, albuminuria, arterial blood pressure and $\mathrm{HbA}_{1} \mathrm{c}$, on the likelihood of obtaining remission during follow-up (data not shown).

During follow-up, the degree of albuminuria was, by definition, lower among patients with remission than those patients without remission (Table 2). In addition, patients with remission had a significantly lower systolic blood pressure and a tendency towards a lower diastolic blood pressure and a lower rate of decline in their GFR than patients without remission. Patients with remission also had significantly lower cholesterol levels, whereas there was no significant difference between the two groups with respect to $\mathrm{HbA}_{1} \mathrm{c}$ and haemoglobin concentrations (Table 2). At the end of follow-up, diabetic retinopathy was present in $18(90 \%)$ of the patients in the remission group and in $57(97 \%)$ of the patients without remission (difference NS).

At the end of the study the mean number of antihypertensive agents used was 3.4 (range two to five) in patients with remission compared with 2.9 (range one to six) in patients without remission (NS). All patients who obtained remission were treated with either an ACE-I or an angiotensin II receptor blocker (ARB), compared with only $46(78 \%)$ of the patients who did not obtain remission $(p<0.05)$. The ACE-I or ARB treatment was initiated before the induction of remission in all patients. The proportion of patients receiving loop diuretics was also greater among patients with remission than among patients without remission $(95 \%$ vs. $75 \%, p<0.05)$, whereas a similar proportion received beta-blockers $(45 \%$ vs $39 \%$, NS), calcium-channel blockers (70\% vs $63 \%$, NS), thiazides ( 15 vs $24 \%$, NS), lipid-lowering drugs $(45 \%$ vs $44 \%, \mathrm{NS})$ or low-dose aspirin ( $75 \%$ vs $63 \%$, NS).

Multiple logistic regression analysis including age, sex, treatment with ACE-I or ARB, and decline of systolic and diastolic blood pressure, cholesterol, and $\mathrm{HbA}_{1} \mathrm{c}$ from baseline to end of follow-up showed that decline in systolic blood pressure and treatment with ACE-I or ARB were
Fig. 1 Proportion of patients obtaining remission according to tertiles of systolic blood pressure reduction from baseline to end of study follow-up $(p<0.05)$. Seventy-nine type 2 diabetic patients with NRA were studied

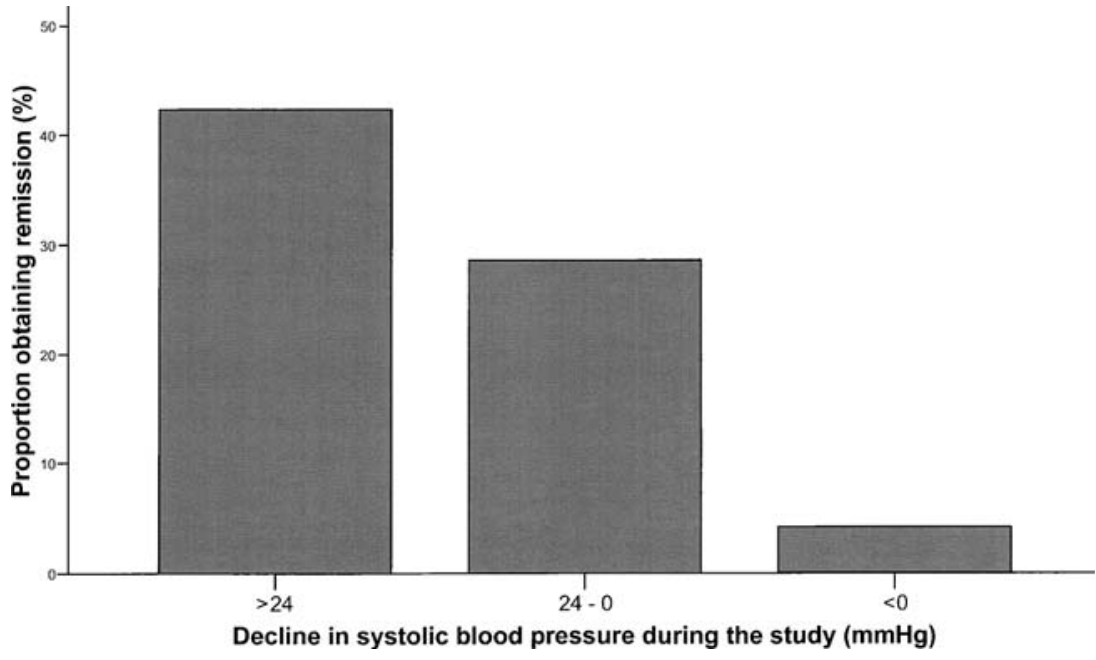


Table 3 Cox proportional hazard model showing impact of remission on the composite endpoint of ESRD or death and on death alone in 79 type 2 diabetic patients with NRA

\begin{tabular}{|c|c|c|c|c|}
\hline \multirow{2}{*}{$\frac{\text { Cox model }}{\text { Estimates }}$} & \multicolumn{2}{|l|}{ ESRD or death } & \multicolumn{2}{|l|}{ Death alone } \\
\hline & $\begin{array}{l}\text { Relative risk } \\
(95 \% \mathrm{CI})\end{array}$ & $p$ value & $\begin{array}{l}\text { Relative risk } \\
(95 \% \mathrm{CI})\end{array}$ & $p$ value \\
\hline \multicolumn{5}{|l|}{ Time-dependent covariate } \\
\hline Remission of NRA & $0.33(0.13-0.90)$ & 0.03 & $0.31(0.12-0.79)$ & 0.01 \\
\hline \multicolumn{5}{|l|}{ Fixed covariates } \\
\hline Age at entry (per 10 years) & $2.11(1.34-3.32)$ & $<0.01$ & $2.34(1.46-3.76)$ & $<0.001$ \\
\hline Sex (female vs male) & $0.40(0.15-1.05)$ & 0.06 & $0.23(0.08-0.65)$ & $<0.01$ \\
\hline $\begin{array}{l}\text { Systolic blood pressure } \\
\text { (per } 10 \mathrm{mmHg} \text { increase) }\end{array}$ & $1.30(1.08-1.58)$ & $<0.01$ & $1.19(0.98-1.43)$ & 0.07 \\
\hline $\mathrm{HbA}_{1} \mathrm{c}(\%)$ & $1.06(0.86-1.30)$ & 0.58 & $1.09(0.87-1.36)$ & 0.44 \\
\hline
\end{tabular}

significantly associated with obtaining remission. The likelihood of remission was increased by $44 \%$ (95\% CI 5$97 \%$ ) for every $10 \mathrm{mmHg}$ decrease in systolic blood pressure over the study period $(p<0.05)$. The relationship between the change in systolic blood pressure over the study period and the proportion obtaining remission is illustrated in Fig. 1 with patients stratified into tertiles of the change in systolic blood pressure during the study.

\section{ESRD and mortality}

Only six of the 20 patients $(30 \%)$ with remission progressed to the composite endpoint of development of ESRD (two patients) or death (four patients), compared with $39(66 \%)$ of the 59 patients without remission (of whom 16 developed ESRD and 23 died $)(p<0.01)$. In the remission group, both patients who developed ESRD subsequently died during follow-up, resulting in a total of six deceased patients in this group (four died from cardiovascular disease, two from ESRD). In patients without remission 14 of the 16 patients with ESRD subsequently died during follow-up, giving a total of 37 deaths in this group $43 \%$ died from cardiovascular disease, $38 \%$ from ESRD, $14 \%$ from cancer and 5\% from unknown causes).

The estimates of the Cox regression analysis for both the composite endpoint of ESRD and death, and for death alone are shown in Table 3. The effect of obtaining remission was strong, with significant reductions of $67 \%$ for risk of ESRD or death and of $69 \%$ for death alone. In addition, age at onset of NRA, sex and baseline systolic blood pressure showed a significant impact on the outcome in these models.

\section{Discussion}

This long-term observational study demonstrates that NRA occurs frequently in patients with type 2 diabetes and nephropathy, and is associated with a high risk of ESRD and premature death, primarily as a result of cardiovascular disease. However, in contrast to previous observations before the general use of blood-pressure-lowering agents, our study reveals that sustained remission of NRA can be obtained in a sizeable fraction of patients by aggressive antihypertensive treatment, in particular with agents blocking the RAAS. Moreover, remission of NRA is associated with a greatly improved cardiovascular risk profile with a decrease in arterial blood pressure, albuminuria and cholesterol levels. Finally, remission of NRA is associated with a substantial reduction in the risk of progressing to ESRD, and with improved survival prospects.

Our results clearly suggest that even at a very late stage of disease, mechanisms underlying disease progression can be modified by active intervention using agents that reduce arterial blood pressure. Apart from antihypertensive treatment, no other major end-organ-saving treatment modality was introduced early enough during the study period to have a significant impact on remission rates in our study. Notably, no significant changes were seen in $\mathrm{HbA}_{1} \mathrm{c}$ over the study period. Furthermore, no sodium or protein restriction was applied and lipid-lowering drugs and lowdose aspirin were not introduced on a routine basis at Steno Diabetes Center until 2002. The substantial decline in cholesterol among patients with remission of NRA is probably a direct consequence of the reduction in albuminuria, rather than an effect attributable to lipid-lowering treatment, which was similar among patients with and without remission.

There is no previous study in type 2 diabetic patients dealing with the incidence of NRA and the effects of remission on renal and cardiovascular outcome. However, both albuminuria and blood pressure are well-established risk factors of poor renal and cardiovascular outcome in diabetic patients. Furthermore, recent clinical trials have demonstrated that the greater the reduction in albuminuria upon initiation of antihypertensive treatment the better the long-term renal and cardiovascular outcome $[5,6,9]$. Our finding that remission was more frequent among patients receiving treatment with either an ACE-I or ARB is in agreement with previous studies demonstrating that agents blocking the RAAS have a greater antiproteinuric effect than other blood-pressure-lowering compounds $[9,10,16,20]$.

In our study, male sex was associated with an increased risk of reaching the composite endpoint of ESRD or death. Since sex does not influence the rate of decline in GFR among patients with diabetic nephropathy [14], this asso- 
ciation was probably attributable to a higher cardiovascular mortality among men, rather than to a faster progression of renal disease. We were unable to demonstrate an association between $\mathrm{HbA}_{1} \mathrm{c}$ and progression to ESRD or allcause mortality. This may in part be because glycaemic control remained relatively poorly controlled throughout the study. However, we have previously demonstrated in larger cohorts of both type 1 and type 2 diabetic patients with diabetic nephropathy that hyperglycaemia is associated with an increased rate of decline in renal function and a decreased survival rate $[13,14,21]$.

To further improve the prognosis and treatment of diabetic renal disease it will be essential to identify factors that explain why some patients respond well to treatment, i.e. obtain remission, whereas others are less responsive and do not remit. Although we do not have systematic data on renal structure it is possible that patients with remission have a more haemodynamic basis to their proteinuria which may be amenable to therapeutic intervention, whereas patients without remission may have more advanced renal structural lesions and therefore are more likely to progress to ESRD and an early death despite treatment. However, there were no demographic, clinical or laboratory differences between the two groups at the onset of NRA. In particular it should be stressed that there were no differences between the two groups with regard to GFR, albuminuria and arterial blood pressure at the onset of NRA. On average, moreover, it took between 2 and 3 years of antihypertensive treatment to induce remission, which does not support the idea of a pure haemodynamic effect. Other factors contributing to differences in therapeutic response may include genetic differences, such as polymorphisms within the RAAS [22], differences in dietary salt intake, inadequate dosing and/or combinations of blood-pressure-lowering agents, and non-adherence to prescribed drugs [23].

In conclusion, our study demonstrates that NRA occurs frequently in patients with type 2 diabetes with nephropathy. However, remission of NRA is feasible in a large proportion of the patients by aggressive antihypertensive treatment. Remission is associated with a greatly improved cardiovascular risk profile and is associated with a substantial reduction of the risk of reaching ESRD and of allcause mortality. Future studies are needed to clarify differences in the responsiveness to treatment among patients with and without remission.

Acknowledgements The Paul and Erna Sehested Hansen Foundation The Per S. Henriksen Foundation, and the Danish Diabetes Association are gratefully thanked for their financial support. We greatly appreciate the help from our laboratory technicians Ulla Meng Smidt, Berit Ruud Jensen, Birgitte Vilsbøll Hansen, Lotte Pietraszek, Tina Ragnholm Juhl, and Inge-Lise Rossing for their help with collecting the data. Furthermore we are indebted to Bendix Carstensen and Peter Rossing for their help with the statistical analysis.

\section{References}

1. Kussman MJ, Goldstein H-H, Gleason RE (1976) The clinical course of diabetic nephropathy. JAMA 236:1861-1863

2. Parving H-H, Mauer M, Ritz E (2004) Diabetic nephropathy. In: Brenner BM (ed) Brenner and Rector's the Kidney. WB Saunders, Philadelphia, pp 1777-1818

3. Watkins PJ, Blainey JD, Brewer DB et al (1972) The natural history of diabetic renal disease. A follow-up study of series of renal biopsies. Q J Med 41:437-456

4. Rossing P, Hommel E, Smidt UM, Parving H-H (1994) Reduction in albuminuria predicts a beneficial effect on diminishing the progression of human diabetic nephropathy during antihypertensive treatment. Diabetologia 37:511-516

5. de Zeeuw D, Remuzzi G, Parving H-H et al (2004) Proteinuria, a target for renoprotection in patients with type 2 diabetic nephropathy: lessons from RENAAL. Kidney Int 65:2309-2320

6. de Zeeuw D, Remuzzi G, Parving H-H et al (2004) Albuminuria, a therapeutic target for cardiovascular protection in type 2 diabetic patients with nephropathy. Circulation 110:921-927

7. Mogensen CE (1976) Progression of nephropathy in long-term diabetics with proteinuria and effect of initial antihypertensive treatment. Scand J Clin Lab Invest 36:383-388

8. Parving H-H, Smidt UM, Friisberg B, Bonnevie-Nielsen V, Andersen AR (1981) A prospective study of glomerular filtration rate and arterial blood pressure in insulin-dependent diabetics with diabetic nephropathy. Diabetologia 20:457-461

9. Brenner BM, Cooper ME, de Zeeuw D et al (2001) Effects of losartan on renal and cardiovascular outcomes in patients with type 2 diabetes and nephropathy. N Engl J Med 345:861-869

10. Lewis EJ, Hunsicker LG, Clarke WR et al (2001) Renoprotective effect of the angiotensin-receptor antagonist irbesartan in patients with nephropathy due to type 2 diabetes. N Engl J Med 345:851-860

11. Herbert LA, Bain RP, Verme D et al (1994) Remission of nephrotic-range proteinuria in type I diabetes. Kidney Int 46:1688-1693

12. Hovind P, Rossing P, Tarnow L, Toft H, Parving J, Parving H-H (2001) Remission of nephrotic-range albuminuria in type 1 diabetic patients. Diabetes Care 24:1972-1977

13. Hovind P, Tarnow L, Rossing P, Carstensen B, Parving H-H (2004) Improved survival in patients obtaining remission of nephrotic range albuminuria in diabetic nephropathy. Kidney Int 66:1180-1186

14. Rossing K, Christensen PK, Hovind P, Tarnow L, Rossing P, Parving H-H (2004) Progression of nephropathy in type 2 diabetic patients. Kidney Int 66:1596-1605

15. American Diabetes Association (1993) Treatment of hypertension in diabetes. Diabetes Care 16:1394-1401

16. Parving $\mathrm{H}-\mathrm{H}$, Lehnert $\mathrm{H}$, Brochner-Mortensen J, Gomis R, Andersen S, Arner P (2001) The effect of irbesartan on the development of diabetic nephropathy in patients with type 2 diabetes. N Engl J Med 345:870-878

17. Bröchner-Mortensen J, Rödbro P (1976) Selection of routine method for determination of glomerular filtration rate in adult patients. Scand J Clin Lab Invest 36:35-45

18. Miles DW, Mogensen CE, Gundersen HJG (1970) Radioimmunoassay for urinary albumin using a single antibody. Scand J Clin Lab Invest 26:5-11

19. Feldt-Rasmussen B, Dinesen B, Deckert M (1985) Enzyme immunoassay: an improved determination of urinary albumin in diabetics with incipient nephropathy. Scand J Clin Lab Invest 45:539-544 
20. Lewis E, Hunsicker L, Bain R, Rhode R (1993) The effect of angiotensin-converting-enzyme inhibition on diabetic nephropathy. N Engl J Med 329:1456-1462

21. Hovind P, Rossing P, Tarnow L, Smidt UM, Parving H-H (2001) Progression of diabetic nephropathy. Kidney Int 59:702-709
22. Jacobsen $\mathrm{P}$, Tarnow L, Carstensen B, Hovind $\mathrm{P}$, Poirier $\mathrm{O}$, Parving H-H (2003) Genetic variation in the renin-angiotensin system and progression of diabetic nephropathy. J Am Soc Nephrol 14:2843-2850

23. Pladevall M (2004) Clinical outcomes and adherence to medications measured by claims data in patients with diabetes. Diabetes Care 27:2800-2805 